\title{
Mycobacterium chelonae
}

National Cancer Institute

\section{Source}

National Cancer Institute. Mycobacterium chelonae. NCI Thesaurus. Code C86536.

A species of aerobic, Gram positive, rods assigned to the phylum Actinobacteria. This species is acid fast, oxidase negative, urease and catalase positive, nonmotile and nonsporulating. M. chelonae is a fast growing mycobacterium isolated from environmental, animal and human sources and is often associated with post-operative soft tissue infection. 